\title{
Lumbosacral Epidural Primitive Neuroectodermal Tumor (PNET): Case Report and Literature Review
}

\author{
Keyvan EGHBAL ${ }^{1}$, Amir Reza DEHGHANIAN ${ }^{2}$, Fariborz GHAFFARPASAND ${ }^{3}$ \\ ${ }^{1}$ Shiraz University of Medical Sciences, Department of Neurosurgery, Shiraz, Iran \\ ${ }^{2}$ Shiraz University of Medical Sciences, Department of Pathology, Shiraz, Iran \\ ${ }^{3}$ Shiraz University of Medical Sciences, Student Research Committee, Department of Neurosurgery, Shiraz, Iran
}

\section{ABSTRACT}

The primitive neuroectodermal tumors (PNETs) of the spine are rare. They are usually intramedullary and reported in children. We herein report an epidural PNET of lumbosacral area presenting with the cauda equina syndrome in an adult. A 38-year-old woman presented to our emergency room with acute onset lower extremity weakness and urinary incontinence. Emergent magnetic resonance imaging revealed a mass lesion isointense on T1-weighted and heterogeneously hyperintense in T2-weighted images in the epidural lumbosacral area. The patient underwent emergent laminectomy of L1-L3 and total resection of the lesion. The patient's neurological examination improved dramatically after the surgery and after 6-month of follow-up, she was neurologically intact. The histopathological and immunohistochemical evaluations revealed PNET. Epidural PNET of the spinal column, although rare, can present with an acute neurological deficit. Surgery remains the treatment of choice and immunohistochemistry is required for confirming the diagnosis.

KEYWORDS: Primitive neuroectodermal tumor, Lumbosacral, Epidural

\section{INTRODUCTION}

$\mathrm{T}$ The primitive neuroectodermal tumors (PNETs) are a group of aggressive tumors with undifferentiated histology (with minimal differentiation toward neural tissue), most commonly detected in children and young adults (9). PNETs of the central nervous system (CNS) have been reported in several locations including the cerebrum, cerebellum, brainstem, pineal gland, spinal cord and peripheral nerves $(9,14,16)$. PNET of the spinal column is a rare condition and most of the reported cases are intramedullary $(4,6,7,15,17)$. Only few cases of isolated epidural PNET have been reported in the literature, most of them in the children $(2,3,10,11)$. In addition, most of the cases present with slowly progressive neurological deficit while only one case has been reported with the cauda equina syndrome (12). We herein report an isolated lumbosacral epidural PNET in a middle-aged adult who presented with cauda equina syndrome.

\section{CASE REPORT}

A 38-year-old lady presented to our emergency room with acute onset progressive weakness of both lower extremities along with urinary incontinency since 5 hours before presentation. She had no positive past medical history except for a 3-month non-radiating low-back pain especially during activity which was associated with muscle cramps and paresthesia in the distal part of both lower extremities. She had previously seen a physician and was followed on physiotherapy and medications such as non-steroidal anti-inflammatory drugs. On physical examination, she had proximal muscle powers of $3 / 5$ and distal muscle powers of $2 / 5$, hypoesthesia in both lower extremities (beneath the inguinal canal) and saddle anesthesia. She was diagnosed with the cauda equina syndrome and underwent emergent magnetic resonance imaging (MRI) of the lumbosacral area. The imaging revealed a round epidural mass lesion compressing the conus medullaris posteriorly (Figure 1A, B). Emergent L1-L3 laminectomy and 
total resection of the mass lesion was carried out (Figure 1C). The microscopic evaluation of the tumor tissue revealed vascular sheets of small round blue cells with a faintly lobular pattern. Tumor cells were arranged in pseudorosette fashion with high mitotic rate. The tumor cells infiltrated skeletal muscular bundles of paravertebral muscle (Figure 2A-F). Immunohistochemical evaluation showed strong diffuse CD99 staining and high proliferative index (Ki-67). Other possibilities such as rhabdomyosarcoma and lymphoma were excluded by negativity of desmin and leukocyte common antigen (LCA), respectively. Accordingly, PNET was diagnosed (Table I). After a week, the muscle powers improved and a 6-month follow-up demonstrated normal muscle powers and normal sphincter tones. Brain and whole spine MRI revealed no other associated lesion and she is now receiving chemotherapy and her neurological examination is intact after 6 months of followup.

\section{DISCUSSION}

Spinal PNET is a rare condition, but the incidence is increasing because of improved diagnostic modalities (13). Several cases of spinal PNET have been reported in the literature, mostly in children $(4,6,7,15)$. However, epidural PNET is an extremely rare presentation with less than 15 cases reported in the literature $(1-3,5,8,10-13)$. We also reported a case of lumbosacral epidural PNET in a 38-year-old woman presenting with the cauda equina syndrome. The patient was successfully managed surgically and restored the muscle powers in early postoperative period. Recently, Sade et al. reported a similar case of epidural PNET presenting with acute neurological deficit in a 6-year-old boy which was also managed surgically (12).
It has been reported that muscular weakness is the most common presentation of patients with spinal PNETs $(13,17)$. However, the primary symptoms of the spinal irritation and compression are considered to be the sensory symptoms including local pain, paresthesia and radiculopathy (13). In patient with extradural PNET, the symptoms mimic any type of disc herniation. In the current case, the patient had a 3-month

Table I: The Immunohistochemistry Profile of the Epidural Mass Lesion

\begin{tabular}{lc}
\hline Immunohistochemical markers & Results \\
\hline Vimentin (VIM3B4) & Positive \\
\hline Cytokeratin (AE1/AE3) & Negative \\
\hline Desmin (D33) & Negative \\
\hline EMA(E29) & Negative \\
\hline LCA(2B11+PD7/26) & Negative \\
\hline MYOD1(5.8A) & Negative \\
\hline CD3 & Negative \\
\hline Pax 5 & Negative \\
\hline MIC2(CD99)(12E7) & Positive \\
\hline TdT & Negative \\
\hline Synaptophysin & Negative \\
\hline Ki-67(MIB-1) & Positive in 40\% of \\
\hline
\end{tabular}
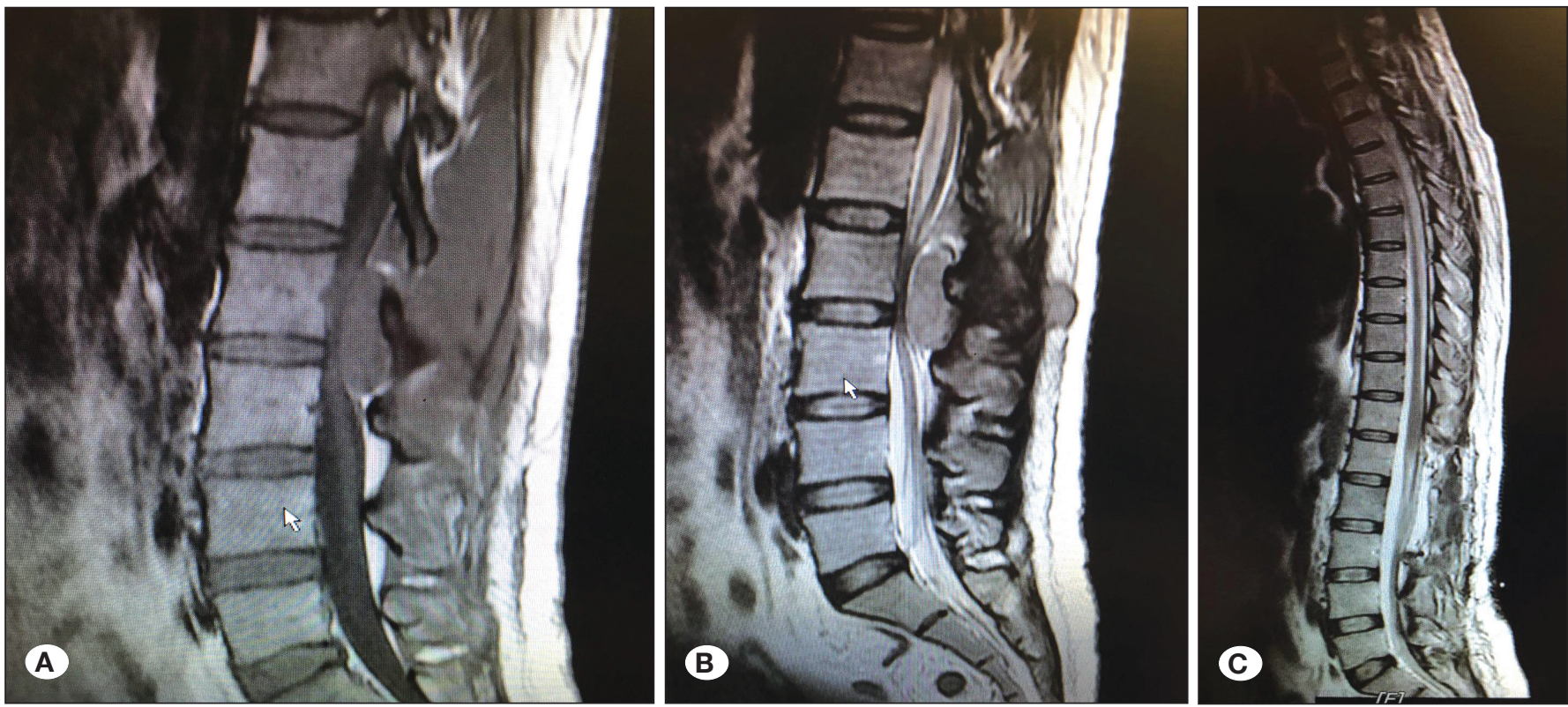

Figure 1: Magnetic resonance imaging (MRI) of the patient demonstrating a round epidural isointense in T1-weighted sagittal (A), and heterogeneously hyperintense in T2-weighted sagittal images (B), lesion compressing the conus medullaris posteriorly (L1-L2 levels); the postoperative T2-weighted sagittal image of the lumbosacral area demonstrating laminectomy of L1-L3 and total excision of the mass resulting in decompression of the conus medullaris and free CSF flow (C). 
history of paresthesia and non-radiating low back pain. She also complained of muscle cramps. These symptoms could be explained with chronic compression and irritation of cauda equina and the conus medullaris. The interesting part of this case was that she presented with cauda equina syndrome which is a very rare presentation only reported once (12). A literature review reveals that more than $50 \%$ of spinal extradural PNETs occur in the lumbar region $(1,5,12)$. Sacral, thoracic and cervical PNETs are rare, especially in the epidural space $(6,8,13)$. According to a recent meta-analysis by Saeednia et al. the median age for spinal PNET is 13 years and there is male predominance (13). The primary spinal PNETs are rarely encountered after the age of 35 years and are common in children $(13,17)$. The current case was a woman with an age of 38 years which makes it very rare. The diagnosis cannot be confirmed by histopathological examination utilizing the routine staining methods. Immunohistochemistry is currently required for confirming the diagnosis (16). It has been demonstrated that CD4+, CD8+, gammadelta-T-cells, and Tregs are found in PNETs. Activation status of cytotoxic cells is variable and the cell surface MHC-I and CD1d expression is downregulated (16). In the current case, strong diffuse CD99 staining was found along with a high proliferative index (Ki-67) while desmin and LCA were negative.

The treatment of the spinal PNETs remains vague and unidentified because of lack of evidence and rarity of the condition. Currently, spinal PNET is treated as other CNS PNETs and extraspinal PNETs. Surgery remains the mainstay of the treatment with the goal of both decompression and diagnosis. The surgery should be performed in a way to remove as much as the tumor and the goal is total resection (17). It has been shown that chemotherapy is associated with improved 1- and 2-year survival rates after total or subtotal tumor resection in both primary intraspinal and CNS PNETs. Thus, it is recommended that all the patients with the diagnosis

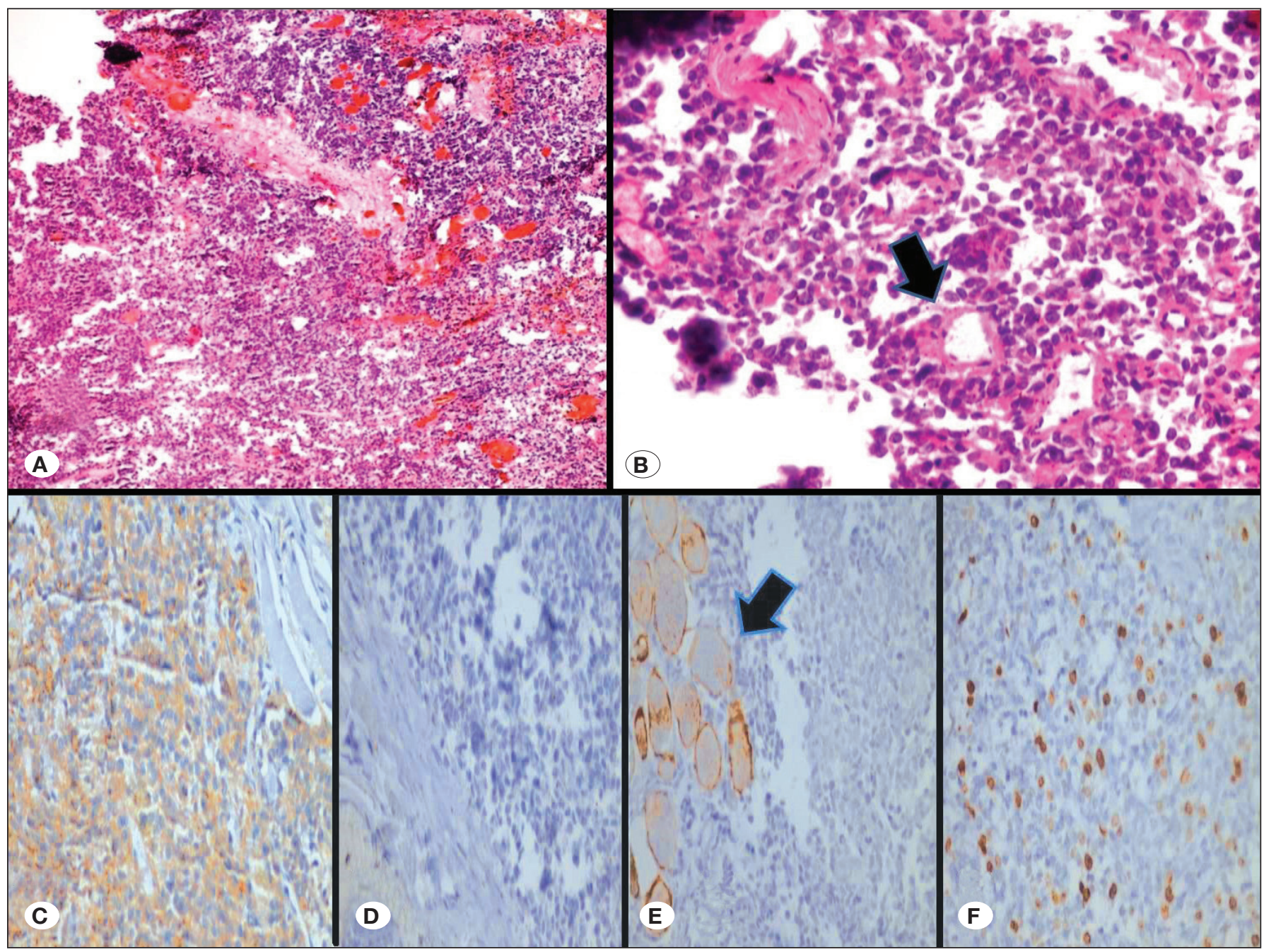

Figure 2: Histopathology of excised lesion: A) Histopathology evaluation shows small round cell tumor with highly vascular stroma (Hematoxylin and Eosin, $\times 200$ ); B) pseudo-rosette formation by tumor cells (Arrow) $\times 400$; C) immunohistochemical evaluation of the tumor shows diffuse strong CD99 positivity $\times 400$; D) leukocyte common antigen (LCA) is negative; E) desmin is negative in tumor cells but stains skeletal muscle bundles (Arrow) $\times 400$; F) proliferative index $(\mathrm{Ki}-67)$ is high $(\times 400)$. 
of spinal PNET receive adjuvant chemotherapy (13). The role of radiotherapy has not been elucidated in the treatment of spinal PNETs.

\section{- CONCLUSION}

Primary lumbar epidural PNET is an extremely rare condition that can present with an acute neurological deficit. Surgery remains the treatment of choice and should be performed in an emergency setting when acute neurological deficit is encountered. Immunohistochemistry is required for confirming the diagnosis.

\section{- REFERENCES}

1. Aydin MV, Sen O, Ozel S, Kayaselcuk F, Caner H, Altinors N: Primary primitive neuroectodermal tumor within the spinal epidural space: Report of a case and review of the literature. Neurol Res 26: 774-777, 2004

2. Chang SI, Tsai MC, Tsai MD: An unusual primitive neuroectodermal tumor in the thoracic epidural space. J Clin Neurosci 17: 261-263, 2010

3. Feng JF, Liang YM, Bao YH, Pan YH, Jiang JY: Multiple primary primitive neuroectodermal tumours within the spinal epidural space with non-concurrent onset. J Int Med Res 36: 366-370, 2008

4. Hrabalek L, Kalita O, Svebisova H, Ehrmann J Jr, Hajduch M, Trojanec R, Kala M: Dumbbell-shaped peripheral primitive neuroectodermal tumor of the spine--case report and review of the literature. J Neurooncol 92: 211-217, 2009

5. Izycka-Swieszewska E, Stefanowicz J, Debiec-Rychter M, Rzepko R, Borowska-Lehman J: Peripheral primitive neuroectodermal tumor within the spinal epidural space. Neuropathology 21: 218-221, 2001

6. Jain A, Jalali R, Nadkarni TD, Sharma S: Primary intramedullary primitive neuroectodermal tumor of the cervical spinal cord. Case report. J Neurosurg Spine 4: 497-502, 2006

7. Khmou M, Malihy A, Lamalmi N, Rouas L, Alhamany Z: Peripheral primitive neuroectodermal tumors of the spine: $A$ case report and review of the literature. BMC Res Notes 9: 438, 2016
8. Kiatsoontorn K, Takami T, Ichinose T, Chokyu I, Tsuyuguchi $\mathrm{N}$, Ohsawa M, Ohata K: Primary epidural peripheral primitive neuroectodermal tumor of the thoracic spine. Neurol Med Chir (Tokyo) 49: 542-545, 2009

9. Lester RA, Brown LC, Eckel LJ, Foote RT, NageswaraRao AA, Buckner JC, Parney IF, Wetjen NM, Laack NN: Clinical outcomes of children and adults with central nervous system primitive neuroectodermal tumor. J Neurooncol 120: 371-379, 2014

10. Musahl V, Rihn JA, Fumich FE, Kang JD: Sacral intraspinal extradural primitive neuroectodermal tumor. Spine J 8: 10241029, 2008

11. Ozdemir N, Usta G, Minoglu M, Erbay AM, Bezircioglu $H$, Tunakan M: Primary primitive neuroectodermal tumor of the lumbar extradural space. J Neurosurg Pediatr 2: 215-221, 2008

12. Sade R, Polat G, Cakir M, Ogul H, Yesilyurt H, Kantarci M: PNET: A rare cause of spinal cord compression. Spine $J 16$ : e437-438, 2016

13. Saeedinia $S$, Nouri $M$, Alimohammadi $M$, Moradi $H$, Amirjamshidi A: Primary spinal extradural Ewing's sarcoma (primitive neuroectodermal tumor): Report of a case and meta-analysis of the reported cases in the literature. Surg Neurol Int 3: 55, 2012

14. Samkari A, Hwang E, Packer RJ: Medulloblastoma/Primitive neuroectodermal tumor and germ cell tumors: The uncommon but potentially curable primary brain tumors. Hematol Oncol Clin North Am 26: 881-895, 2012

15. Sharma P, Das KK, Mehrotra A, Srivastava AK, Sahu RN, Jaiswal A, Pandey R, Behari S, Bhaisora KS, Sardhara $\mathrm{J}$ : Cervicomedullary intramedullary peripheral primitive neuroectodermal tumor with intratumoral bleed: Report of one case and review of literature. J Craniovertebr Junction Spine 7: 111-114, 2016

16. Vermeulen JF, van Hecke W, Spliet WG, Villacorta Hidalgo J, Fisch P, Broekhuizen R, Bovenschen N: Pediatric primitive neuroectodermal tumors of the central nervous system differentially express granzyme inhibitors. PLoS One 11: e0151465, 2016

17. Yan Y, Xu T, Chen J, Hu G, Lu Y: Intraspinal Ewing's sarcoma/ primitive neuroectodermal tumors. J Clin Neurosci 18: 601606, 2011 